\title{
VACUOLAR ENCEPHALOMYELOPATHY IN MICE INDUCED BY INTRACEREBRAL INOCULATION WITH A CORONAVIRUS JHM-CC STRAIN
}

\author{
Tetsuro Tsukamoto*, Yuzo Iwasaki**, Norio Hirano***, \\ Satoru Haga*** \\ *Department of Neurology, Fukushima Medical College, Fukushima,, Sendai, Japan \\ **Department of Neurological Sciences, Tohoku University School of Medicine \\ Sendai, Japan \\ $* * *$ Department of Veterinary Microbiology, Iwate University, Morioka, Japan
}

\section{INTRODUCTION}

JHM-CC virus, a mutant strain of mouse hepatitis virus, was isolated by Hirano in 1980 from a DBT cell culture persistently infected with the neurovirulent strain, JHM ${ }^{1}$. This mutant strain is characterized by formation of smaller plaques on the DBT cells and by lower cell virulence than the original $\mathrm{JHM}^{1}$. Although several murine coronaviruses such as JHM are known to cause demyelinating encephalitis 2,4 , we found that JHM-CC does not induce primary demyelination, but instead produces characteristic vacuolar degeneration in the brain and spinal cord. In this report we describe the clinical manifestations, neuropathological changes and viral antigen distribution in the central nervous system (CNS) of ICR mice infected intracerebrally with JHM-CC.

\section{MATERIALS AND METHODS}

A total of 107 four-week old ICR mice under periodical surveillance for antibody to MHV were inoculated intracerebrally with $0.02 \mathrm{ml}$ of JHM-CC virus suspension $\left(1 \times 10^{6}\right.$ PFU). Twenty mice were maintained for two months after inoculation to observe any clinical manifestations.

For virus titration, 3 mice were sacrificed every day from 1 to 10 days after inoculation and on days 12 and 14. Individual 10\% tissue homogenates (w/v) in MEM were prepared from the brain, spinal cord, liver and spleen. The infectivity of each tissue homogenate diluted in MEM was assayed by plaqueing in DBT cell culture as reported previously5,6.

For histopathology and viral antigen detection, 3 mice were sacrificed and perfusionfixed with a $10 \%$ buffered formalin solution on days $1,2,3,4,5,7,10,14,21,28,30$, and 2,4 , and 6 months after inoculation. Multiple $3 \mu \mathrm{m}$ paraffin sections were prepared from the brain and spinal cord and were stained with hematoxylin-eosin and by the Kluver-Barrera and Bodian methods. For free-floating preparations, the formalin fixed tissues immersed in a PBS solution containing $20 \%$ sucrose and $0.1 \%$ dimethylsulfoxide, were rapidly frozen in liquid nitrogen and then cut into $40 \mu \mathrm{m}$ thick sections with a cryostat. An immunohistochemical study was carried out on paraffin and free-floating sections by the avidin-biotin peroxidase complex $(\mathrm{ABC})$ method using anti-JHM antibody raised in rabbits.

On days 3, 6 and 9 after inoculation, 3 mice each were fixed by perfusion with $4 \%$ paraformaldehyde and $0.5 \%$ glutaraldehyde in Millonig's phosphate buffer for electron microscopy. The tissues were postfixed with $1 \% \mathrm{OsO}_{4}$, dehydrated and embedded in epoxy resin. Ultrathin sections were stained with uranyl acetate and lead citrate and examined with a Hitachi H 600 electron microscope. 

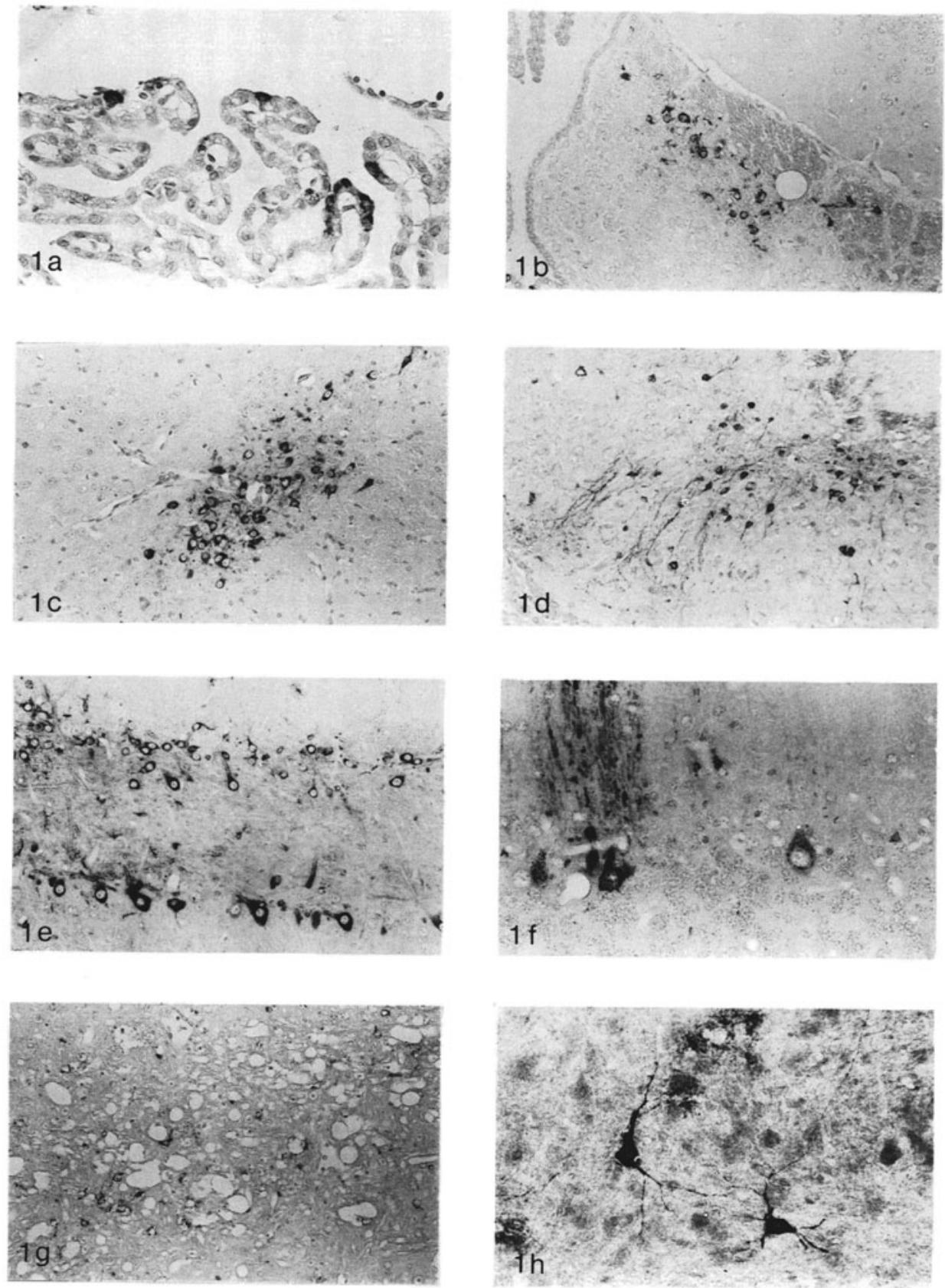

Fig. 1. Immunohistochemistry for JHM-CC viral antigen distribution in the CNS. a-g: $3 \mu \mathrm{m}$ thick paraffin sections. h: $40 \mu \mathrm{m}$-thick floating sections. a. Choroidal plexus, one day post inoculation (p.i.). b. Habenular nucleus, 2 days p.i. c. Piriform cortex, 2 days p.i. d. Cingulate cortex, 3 days p.i. e. Olfactory bulb, 43 days p.i. f. Cerebellum, 4 days p.i. Purkinje cells and small cells in the molecular layer are positive. g. Brainstem, 7 days p.i. Vacuolar degeneration are progressing, whereas viral antigens are diminishing, h. Spinal cord neurons, 7 days p.i. 


\section{RESULTS}

Close clinical observation of 20 mice showed all of them to be irritable and hypersensitive 5 to 7 days after inoculation. Two mice developed hind leg paresis on day 8 and 3 more on day 9 . Four of 5 paralysed mice recovered from the disease after 15 days, whereas one mouse died of severe paresis on day 22 . The other animals showed no symptoms.

Hindlimb paresis also developed 7 to 9 days after inoculation in about $20 \%$ of the mice designated for virus titration and for histopathology. A few severely paralysed animals died from difficulty in feeding. Even in the mice without clinical symptoms, the virus was constantly recovered from the brain and spinal cord during the first two weeks of the infection. The highest titer of the virus was recovered from the mice sacrificed on day 4 or $5\left(10^{6}\right.$ PFU/0.2g). The titer declined gradually and became negative on day 14 . Infectious virus was never recovered from the liver or spleen.

An immunohistochemical study revealed a spatial sequence for the progression of the viral infection in the CNS. On day 1, viral antigen positive cells were sporadically found in the ependyma, choroid plexus and corpus callosum (Fig.1a). On day 2, several neuronal cells in the habenular nucleus, piriform cortex and entorhinal cortex became positive for viral antigen (Fig.1b,c). By day 3, the infection had apparently spread to the cingulate cortex, accumbens nucleus, subiculum, caudate-putamen, thalamus, hypothalamus, substantia nigra, septal nucleus and even to the lower brain-stem (Fig.1d). On day 4, the viral antigens were
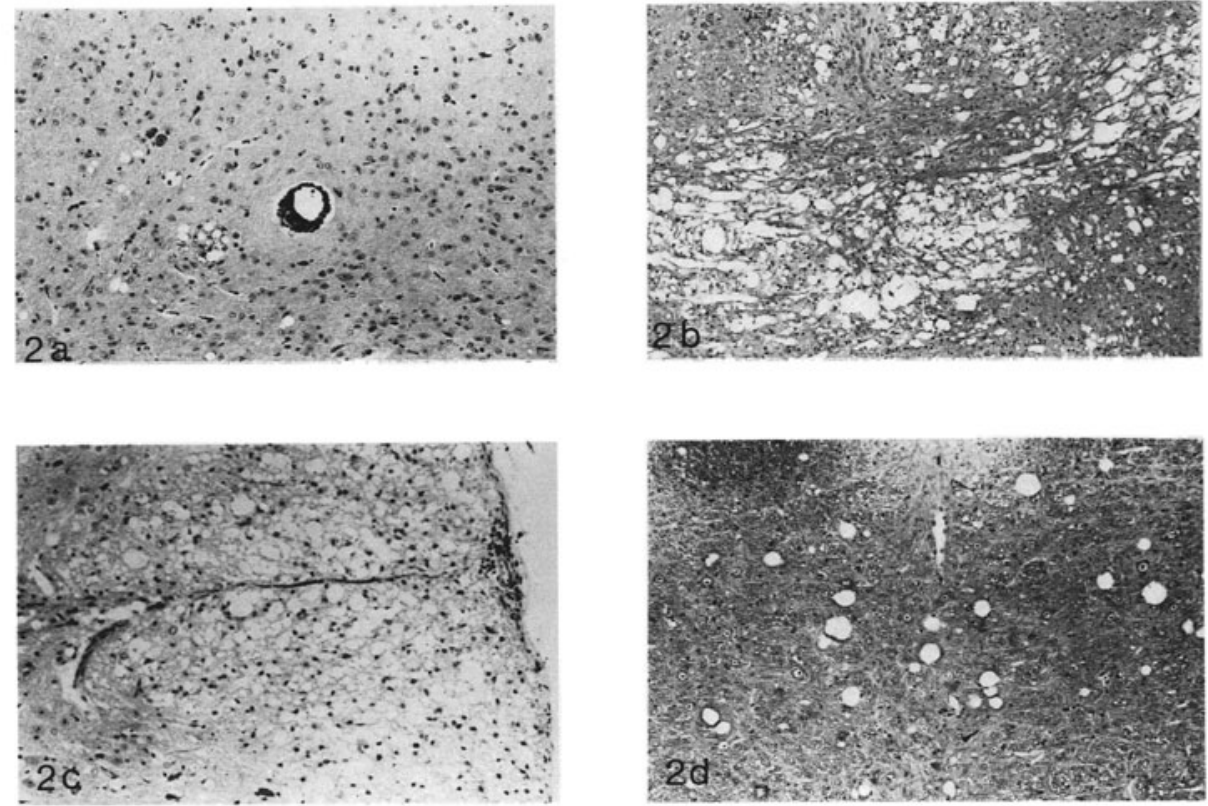

Fig. 2. Histopathology in paraffin sections stained with H-E. a. Perivascular cell infiltration in the pons, 28 days p.i. b. Marked vaculation in the pons with the paucity of inflammatory changes, 18 days p.i. c. Marked vacuolar changes in the spinal cord and subarachnoid inflammatory cell infiltration, 28 days p.i. d. Smaller vacuoles in the pons 2 months p.i. These possibly reflect an ongoing reparative process of vacuolation. 


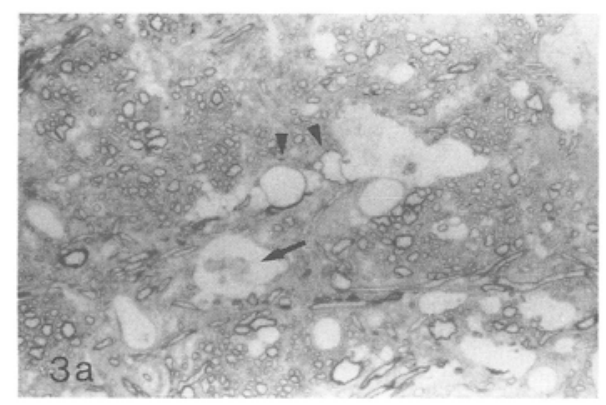

Fig. 3a. Vacuolar changes on day 9 p.i. One- $\mu$ m plastic section of pons stained with toluidine blue. Myelin splitting (arrow heads) and axonal degeneration (arrow) are seen.

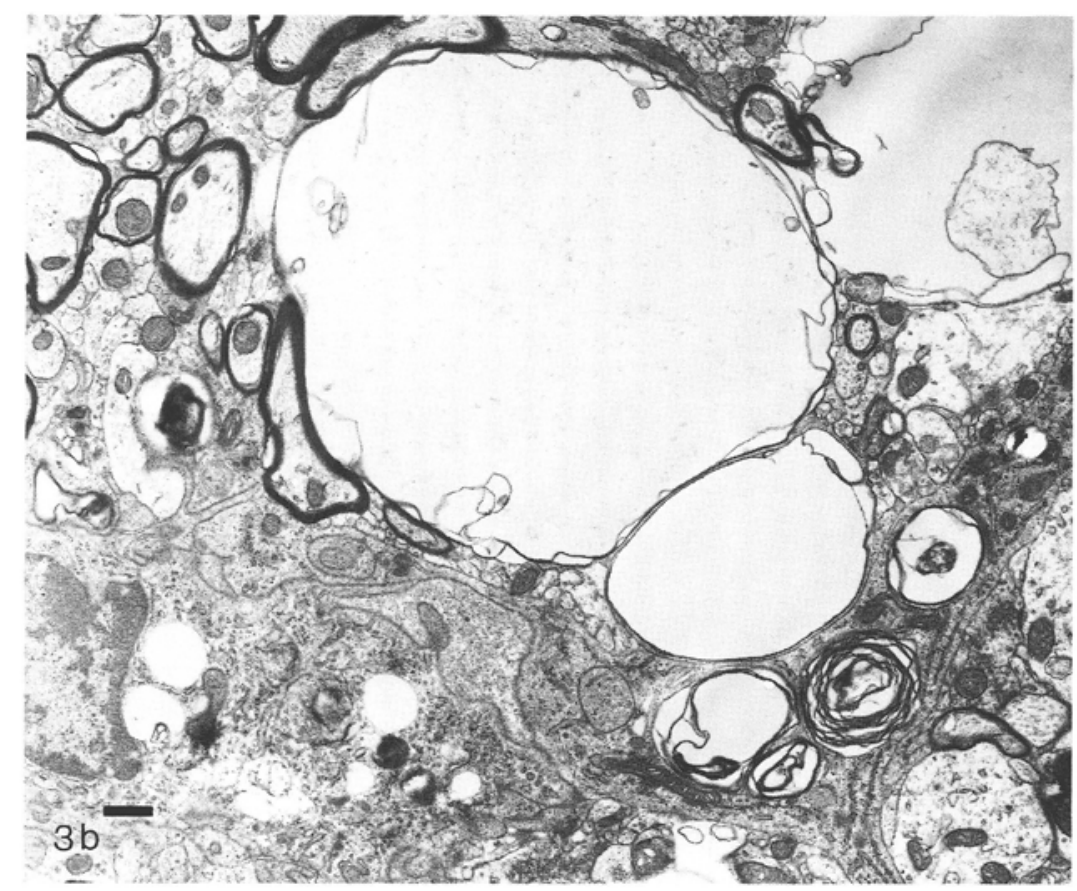

Fig. 3b. Ultrastructure of vacuolar changes on day 9 p.m. Myelin splitting and periaxonal edema. bar $=1 \mu \mathrm{m}$.

demonstrable in the mitral cells and other neurons in the olfactory bulb, and occasionally in the Purkinje and granular cells of the cerebellum and spinal cord neurons (Fig.1e-g). The viral antigens were most strongly and widely expressed on day 5 when the antigens were almost exclusively localized to neurons and rarely to glial cells (Fig.1b). The viral antigens in the CNS gradually diminished and became undetectable by day 14 .

Pathologically, inflammatory changes were rather inconspicuous. Mild mononuclear cell infiltration in the subarachnoid and perivascular spaces was noted on day 2 after inoculation. Between days 3 and 5, necrotic neurons were sporadically seen in the periaqueductal region, 


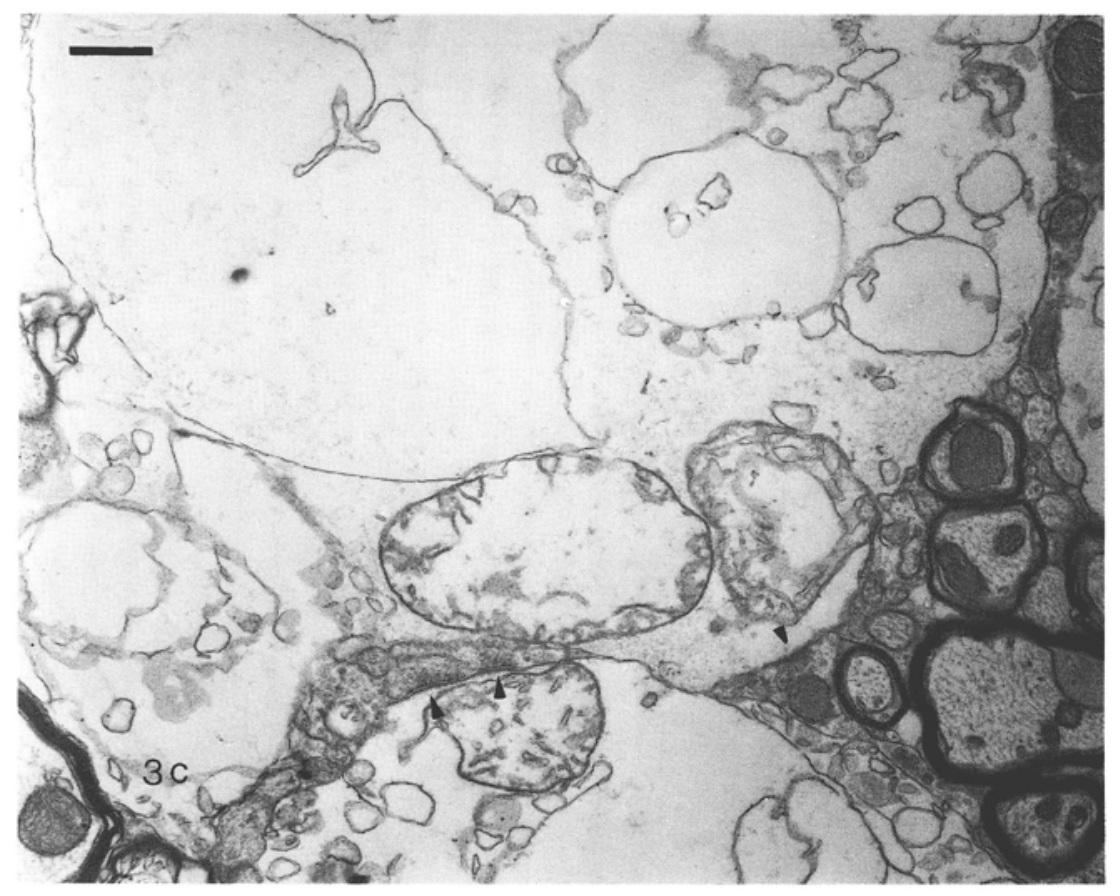

Fig. 3c. Ultrastructure of vacuolar changes in pons. Swollen axons containing bulging mitochondria and membrane-lined vacuoles. Synaptic vesicles are indicated by arrow heads. bar $=1 \mu \mathrm{m}$.

pontine tegmentum and anterior horns of the spinal cord, often without inflammatory cell reaction. Mild ependymitis was evident in some mice. On day 7 , severe neuronal degeneration developed in the olfactory bulb and lobe, and they often became totally necrotic. In other parts of the brain and spinal cord, however, such destructive changes were rare, and foci of microglial proliferation and perivascular mononuclear cell infiltration were sparsely distributed in the cerebral cortex and paraventricular tissues (Fig.2a). These inflammatory changes were most florid in the tissue examined on day 14.

In contrast to the relative paucity of inflammatory changes, the development of vacuolar or spongiform changes was impressive. Small foci of tissue vacuolation were first noted on day 4. These vacuolar changes progressed in size and number even after disappearance of the infectious virus and viral antigens from the CNS tissue and were most conspicuous in the mice sacrificed between days 14 and 28. Although numerous vacuoles of varying size were randomly distributed in the cingulate cortex, corpus callosum, basal ganglia, thalamus, internal capsule, midbrain and cerebellum, the brainstem and the spinal gray and white matter were most extensively involved (Fig.2b,c). Despite the presence of lymphocytes and some plasma cells in the subarachnoid space and thick perivascular cuffs around the parenchymal vessels, macrophages were almost completely absent at the sites of vacuolar degeneration. Following Kluver-Barrera and Bodian staining, the vacuolation did not appear to be related to either primary demylination or axonal swelling. After 2 months foci of vacuolar changes became smaller and decreased in number with the margins somewhat blurred and after 4 months they were no longer observed.

Electron microscopic observations disclosed that vacuolar degeneration was largely attributable to widening of the periaxonal spaces and intra-lamellar edema in the myelin sheath (Fig.3a,b). In addition, swollen axons with bulging mitochondria and membrane-lined vacuoles were occasionally seen (Fig.3c). There were, however, no evidence for primary demyelination. Viral particles were only rarely observed in neuronal cells. 


\section{DISCUSSION}

In contrast to fulminant encephalitis induced by the parental strain of JHM, the intracerebral infection with JHM-CC virus 7,8 , a mutant strain of mouse hepatitis virus (JHM), resulted only in mild hindlimb paresis in some mice or no symptoms in others. Irrespective of clinical manifestations, however, all mice developed histopathological lesions and viral antigens in the CNS. The most distinctive pathological findings were spongiform changes or severe vacuolization in the brain and spinal cord developing relatively delayed after early appearance of mild inflammatory cell infiltration in the subarachnoidal and perivascular spaces.

The progression of these vacuolar changes did not parallel chronologically the sequence of infectious virus levels and the intensity and extension of the viral antigens in the CNS, but these seemed anatomically to be related to the sites of viral replication. They increased in size and number where the viral antigens had been expressed immunohistochemically, even after disappearance of the infectious virus and viral antigens. It is worth noting, however, that vacuolar degeneration was no longer seen 4 months after inoculation. This could be suggestive of reversibility of the vacuolar changes. Furthermore, in the mice sacrificed 2 months after inoculation, vacuolar changes were less conspicuous than those in the mice sacrificed earlier. Smaller vacuoles with a somewhat obscure inner margin might reflect the ongoing reparative process of vacuolation.

Ultrastructural studies showed that these vacuolar changes were not primary demyelination, but were mostly attributable to periaxonal edema, intramyelinic splits and swollen axons containing degenerated mitochondria and membrane-lined vacuoles.

Similar spongiform or vacuolar changes have been found in some retrovirus infections ${ }^{9-12}$. Elucidation of the mechanism of vacuolar degeneration in this JHM-CC infection model could provide a better understanding of new types of neurological disorders associated with viral infections including vacuolar myelopathy in AIDS 11,12 .

\section{ACKNOWLEDGEMENT}

This study was supported by a grant-in-aid for scientific research from the Ministry of Education, Science and Culture, Tokyo, Japan (61570381).

\section{REFERENCES}

1. N. Hirano, K. Fujiwara, S. Hino and M. Matumoto. Persistent infection with mouse hepatitis virus, JHM strain, in DBT cell culture. In: "Biochemistry and Biology of Coronaviruses", V. ter Meulen, S.Siddell and H. Wege eds., Plenum Publishing Corp., New York, 301-308 (1981).

2. L.P. Weiner. Pathogenesis of demyelination induced by a mouse hepatitis virus (JHM virus). Arch.Neurol. 28: 298-303 (1973).

3. S.A. Stohlman and L.P. Weiner. Chronic central nervous system demyelination in mice after JHM virus infection. Neurology (N.Y.) 31: 38-44 (1981).

4. R. Watanabe, H. Wege and V. ter Meulen. Comparative analysis of coronavirus JHMinduced demyelinating encephalomyelitis in Lewis and Brown Norway rats. Lab. Invest. 57: 375-384 (1987).

5. N. Hirano, K. Fujiwara, S.Hino and M. Matumoto. Replication and plaque formation of mouse hepatitis virus (MHV-2) in mouse cell line DBT culture. Arch. Gesamte Virusforsch.44: 298-302 (1978).

6. N. Hirano, T. Murakami, K.Fujiwara and M. Matumoto. Utility of mouse cell line for propagation and assay of mouse hepatitis virus. Jpn. J. Exp. Med.48: 71-75 (1978).

7. F.S. Cheever, J.B. Daniels, A.M. Pappenheimer and O.T. Bailey. A murine virus (JHM) causing disseminated encephalomyelitis with extensive destruction of myelin: I. Isolation and biological properties of the virus. J. Exp. Med. 90: 181-194 (1949).

8. N. Hirano, T.Murakami, F. Taguchi,K. Fujuwara and M.Matumoto. Comparison of mouse hepatitis virus strains for pathogenicity in weanling mice infected by various routes. Arch. Virol. 70: 69-73 (1981). 
9. B.R. Brooks,J.R. Swarz and R.T. Johnson. Spongiform polioencephalomelopathy caused by a murine retrovirus. I. Pathogenesis of infection in newborn mice. Lab. Invest. 43: 480-486 (1980).

10. J.R. Swarz, B.R. Brooks and R.T. Johnson. Spongiform polioencephalomyelopathy caused by a murine retrovirus. II. Ultrastructural localization of virus replication and spongiform changes in the central nervous system. Neuropathol. Appl, Neurobiol. 7: 365-380 (1981).

11. C.K.Petito, B.A. Navia, E-S. Cho, B.D. Jordan, D.C. George and R.W. Price. Vacuolar myelopathy pathologically resembling subacute combined degeneration in patients with the acquired immunodeficiency syndrome. N. Engl. J. Med. 312: 874-879 (1985).

12. L.R. Sharer, L.G. Epstein, E-S. Cho and C.K. Petito. HTLV-III and vacuolar myelopathy. N. Engl. J. Med. 315: 62-63 (1986). 